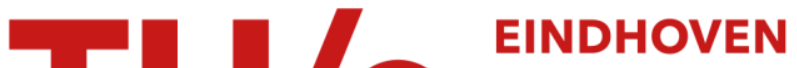 UNIVERSITY OF TECHNOLOGY
}

\section{Flow-induced correlation effects within a linear chain in a polymer melt}

\section{Citation for published version (APA):}

Stepanyan, R., Slot, J. J. M., Molenaar, J., \& Tchesnokov, M. A. (2005). Flow-induced correlation effects within a linear chain in a polymer melt. Physical Review E - Statistical, Nonlinear, and Soft Matter Physics, 72(5), 051807-1/12. [051807]. https://doi.org/10.1103/PhysRevE.72.051807

DOI:

10.1103/PhysRevE.72.051807

Document status and date:

Published: 01/01/2005

\section{Document Version:}

Publisher's PDF, also known as Version of Record (includes final page, issue and volume numbers)

\section{Please check the document version of this publication:}

- A submitted manuscript is the version of the article upon submission and before peer-review. There can be important differences between the submitted version and the official published version of record. People interested in the research are advised to contact the author for the final version of the publication, or visit the $\mathrm{DOI}$ to the publisher's website.

- The final author version and the galley proof are versions of the publication after peer review.

- The final published version features the final layout of the paper including the volume, issue and page numbers.

Link to publication

\section{General rights}

Copyright and moral rights for the publications made accessible in the public portal are retained by the authors and/or other copyright owners and it is a condition of accessing publications that users recognise and abide by the legal requirements associated with these rights.

- Users may download and print one copy of any publication from the public portal for the purpose of private study or research.

- You may not further distribute the material or use it for any profit-making activity or commercial gain

- You may freely distribute the URL identifying the publication in the public portal.

If the publication is distributed under the terms of Article 25fa of the Dutch Copyright Act, indicated by the "Taverne" license above, please follow below link for the End User Agreement:

www.tue.nl/taverne

Take down policy

If you believe that this document breaches copyright please contact us at:

openaccess@tue.nl

providing details and we will investigate your claim. 


\title{
Flow-induced correlation effects within a linear chain in a polymer melt
}

\author{
R. Stepanyan, ${ }^{1}$ J. J. M. Slot, ${ }^{1,2}$ J. Molenaar, ${ }^{3,4}$ and M. Tchesnokov ${ }^{4}$ \\ ${ }^{1}$ Department of Applied Physics, University of Twente, P.O. Box 217, 7500AB Enschede, The Netherlands \\ ${ }^{2}$ Material Science Centre, DSM Research, P.O. Box 18, 6160 MD Geleen, The Netherlands \\ ${ }^{3}$ Faculty of Mathematics and Computer Science, Eindhoven University, P.O. Box 513, 5600 MB Eindhoven, The Netherlands \\ ${ }^{4}$ Department of Applied Mathematics, University of Twente, P.O. Box 217, 7500AB Enschede, The Netherlands
}

(Received 27 June 2005; revised manuscript received 31 August 2005; published 30 November 2005)

\begin{abstract}
A framework for a consistent description of the flow-induced correlation effects within a linear polymer chain in a melt is proposed. The formalism shows how correlations between chain segments in the flow can be incorporated into a hierarchy of distribution functions for tangent vectors. The present model allows one to take into account all the major relaxation mechanisms. Special cases of the derived set of equations are shown to yield existing models and shed some light on the connection between them. Consequences of several assumptions widely used in the literature are analyzed within the developed framework.
\end{abstract}

DOI: 10.1103/PhysRevE.72.051807

PACS number(s): 83.80.Sg, 83.10.Kn, 83.10.Gr

\section{INTRODUCTION}

The rheological properties of monodisperse highmolecular-weight polymer melts have received a lot of attention [1-3]. From the theoretical side, one of the most important achievements in this area is the reptation model proposed more than three decades ago [4], which visualizes microscopic motion of a chain in a melt surprisingly well. This relatively simple picture of molecular relaxation served as a basis for the Doi-Edwards (DE) model [2]. The latter, given its simplicity, describes both the linear and shear thinning flow regimes quite successfully. One of its central assumptions is the existence of an effective tube around a test chain in a polymer melt: a tube comprises all the obstaclesentanglements-imposed by the surrounding chains and restricts the molecular motion to one-dimensional diffusion, which is taken as the major relaxation mechanism.

Despite all its successes, the DE theory failed to reproduce certain rheological properties of melts. In turn, modifications of the original theory have been proposed to remove some of its shortcomings: contour length fluctuations have been introduced $[5,6]$ to explain the 3.4 power law observed for the molecular weight dependence of the zero shear rate viscosity; chain stretch and constraint release have been incorporated [7-9] in order to improve the predictions for fast flows. Among these improvements, the constraint release (CR) relaxation mechanism is conceptually very important, because it allows motion of the chain segments "perpendicular" to the tube if one or more entanglements are removed. $\mathrm{CR}$ arising from the thermal motion of the surrounding chains was mentioned by Doi and Edwards, but its effect was estimated to be negligible in monodisperse system. However, as first described by Marrucci [8], another type of CR occurs in flowing melts. The tube is affinely deformed by the flow, but the chain itself tends to retain its original length by continually retracting within the tube. While slipping along itself, it may release some constraints on the chains around it, which, in turn, get more freedom to move. This relaxation mechanism, convective CR (CCR), is very important for the nonlinear response of polymer melts: one of its great successes is the elimination of the unphysical maximum in the stress-rate curves predicted by DE.
Following these ideas and taking into account the relaxation mechanisms mentioned above, several nonlinear constitutive models have been formulated independently from each other. The ones by Ianniruberto and Marrucci [10] and Mead et al. [11] are based on a formalism for a single-chain orientation tensor and tube survival probability function, respectively, and prescribe how the terminal relaxation time should be adjusted to take the CCR into account. Alternatively, more sophisticated constitutive models originate from the McLeish's group [12-14]: an approach based on a socalled tangent vector correlation function is proposed. Unfortunately, the connection between these theories is poorly established. While based on very similar ideas and giving predictions which are quite close to each other, they differ considerably in the mathematical formalism used. Besides that, each of the models contains some specific assumptions which often cannot be verified within that model itself. Thus, there is a need for a theory in which these assumptions either are not present or can be verified.

One of the important assumptions introduced by DE and then widely used in the literature $[2,7-11,15]$ is the one about the independent orientation of the chain segments even in the presence of flow [2]. At the same time, one of the known flaws of the DE relaxation model is that it predicts the stress to be a decreasing function of the rate in fast flowsbehavior never observed in experiments. This artifact of the theory appears because reptation alone is not effective enough to relax the stress in fast flows. Therefore, in the DE theory, chains become strongly aligned in the flow direction and the melt viscosity drops catastrophically. Introducing CCR helps to "misalign" the chains and avoid this effect, although, as, e.g., a calculation of the extinction angle [11] shows, polymer molecules are still oriented quite strongly. Hence, the hypothesis about independent orientation of different segments along the tube holds in equilibrium (without flow) but certainly breaks down under flow conditions, because of the alignment mentioned above. How to get rid of this inconsistency and avoid the unnecessary and questionable assumptions is one of the questions we strive to answer in the present work. 


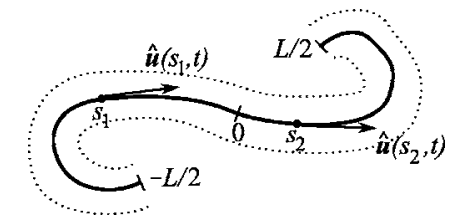

FIG. 1. A primitive path of the length $L$ (solid line) in its tube.

The main goal of this work is to build up a microscopic framework incorporating some of the existing theories of nonlinear polymer dynamics and, at least in principle, allowing for a more detailed description. In the next section, we start from a generalization of the DE theory based on a many point distribution function [16]. We restrict ourselves to inextensible chains, and we will try to avoid introducing any additional hypothesis concerning correlations in orientation of the segments in the derivation of the model. After the master set of equations has been derived, we focus on the analysis of some special cases that lead to already existing theories. This allows us to clarify the relation between them as well as discuss the influence of various assumptions on the final predictions.

\section{MANY-POINT DISTRIBUTION FUNCTION}

In the framework of the tube model [2,3], a linear polymer chain in a melt is described in terms of a primitive path, which is the shortest line connecting the chain's ends and having the same topology as the chain itself. The motion of the primitive path is highly restricted due to presence of the surrounding chains, which create constraints called entanglements and build up an effective tube around the test chain. In what follows, we assume the primitive path to have constant length $L$ : this restricts the formalism to the moderate-flow regime with a typical rate lower than the inverse Rouse (length relaxation) time of the chain.

The primitive path trajectory is completely described by the function $\hat{\boldsymbol{R}}(s, t)$, which gives the spatial position of the point $s$ along the path at the time $t$ (here and further on all the microscopic stochastic quantities are denoted by a caret). We choose the origin of the curvilinear coordinate $s$ in the middle of the path so that $-L / 2 \leqslant s \leqslant L / 2$ and $s= \pm L / 2$ correspond to the chain's ends, Fig: 1 . However, it is more convenient to work with the tangent vector $\hat{\boldsymbol{u}}(s, t) \equiv \partial \hat{\boldsymbol{R}} / \partial s$. E.g., in the original DE theory, the microscopic description of the primitive path dynamics is based upon a stochastic equation for $\hat{\boldsymbol{u}}(s, t)$ (see [2], p. 276, for the derivation):

$$
\begin{aligned}
\hat{\boldsymbol{u}}(s, t+\Delta t)= & \hat{\boldsymbol{u}}(s+\Delta \zeta, t)+\Delta t[\boldsymbol{K} \cdot \hat{\boldsymbol{u}}(s, t) \\
& \left.+\frac{\partial}{\partial s}\left(\hat{\boldsymbol{u}}(s, t) \int_{0}^{s} \mathrm{~d} x \hat{\xi}(x, t)\right)\right] .
\end{aligned}
$$

Each term in Eq. (1) represents a certain type of motion of the path inside its tube. The simplest of these is convectionthe deformation of the tube by the flow applied with the velocity gradient tensor $\boldsymbol{K} \equiv \boldsymbol{\nabla} \boldsymbol{v}$. It deforms the tube affinely and gives rise to the first term in the square brackets. Because the chain strives to preserve its length, it retracts inside the tube. As can be shown, the retraction yields the last term in Eq. (1) with the retraction rate $\hat{\xi}=-\boldsymbol{K}: \hat{\boldsymbol{u}} \hat{\boldsymbol{u}}$ fixed by the constant contour length condition. Finally, the onedimensional diffusion of the entire chain along its own contour-reptation-is responsible for the $\Delta \zeta$ term on the right-hand side (RHS) of Eq. (1). This is the key ingredient in the consideration by [2]: it allows the chain to escape its deformed tube and relax the stress. Mathematically, the displacement $\Delta \zeta$ of the path due to reptation is expressed as a zero-mean Gaussian noise term with $\left\langle\Delta \zeta^{2}\right\rangle=2 D_{\mathrm{c}} \Delta t$, where the one-dimensional diffusivity $D_{\mathrm{c}}$ is related to the disengagement time $\tau_{\mathrm{d}}$ - the characteristic time needed to escape from the original tube-via $D_{\mathrm{c}}=L^{2} /\left(\pi^{2} \tau_{\mathrm{d}}\right)$.

In practice, all the quantities of interest can be expressed in terms of averages of $\hat{\boldsymbol{u}}(s, t)$. For instance, the orientation tensor $\boldsymbol{S}(s, t)$ defined as $\boldsymbol{S}(s, t)=\langle\hat{\boldsymbol{u}}(s, t) \hat{\boldsymbol{u}}(s, t)\rangle$ is the second moment of $\hat{\boldsymbol{u}}$ with respect to averaging over the ensemble of chains. The polymeric contribution to the stress tensor is in its turn expressed in terms of $S$ :

$$
\boldsymbol{\sigma}(t)=\frac{G_{0}}{L} \int_{-L / 2}^{L / 2} \mathrm{~d} s \boldsymbol{S}(s, t),
$$

where $G_{0}$ is the elastic modulus [2]. More complex characteristics, such as a single-chain structure factor $S(\boldsymbol{q}, t)$ measured in neutron scattering experiments [17], can be mapped to the second moments of $\hat{\boldsymbol{u}}$ as well:

$$
\begin{aligned}
S(\boldsymbol{q}, t)= & \int_{-L / 2}^{L / 2} \mathrm{~d} s \int_{-L / 2}^{L / 2} \mathrm{~d} s^{\prime} \\
& \times \exp \left(-\sum_{\alpha, \beta} \frac{q_{\alpha} q_{\beta}}{2} \int_{s}^{s^{\prime}} \mathrm{d} s_{1} \int_{s}^{s^{\prime}} \mathrm{d} s_{2}\left\langle\hat{u}_{\alpha}\left(s_{1}, t\right) \hat{u}_{\beta}\left(s_{2}, t\right)\right\rangle\right) .
\end{aligned}
$$

In principle, the equations for the averages present in Eqs. (2) and (3) can be obtained directly from the stochastic equation (1). However, in many cases, it turns out to be more convenient to derive an equation for the distribution function $f(\boldsymbol{u}, s ; t)$ defined in [2] via the identity

$$
f(\boldsymbol{u}, s ; t)=\langle\delta[\boldsymbol{u}-\hat{\boldsymbol{u}}(s, t)]\rangle .
$$

The function $f$ gives the probability to find the tangent vector $\hat{\boldsymbol{u}}(s, t)$ in the direction $\boldsymbol{u}$ in a thermodynamically large ensemble of chains. In Eq. (4) the angular brackets denote the ensemble average and $\delta$ is the Dirac delta function.

Here we shall not repeat the derivation by Doi and Edwards but only emphasize one of its important shortcomings. Assuming that the dynamics of a primitive path is described by only one function $f(\boldsymbol{u}, s ; t)$, they totally ignore any possible correlations along the chain so that the segments $s_{1}$ and $s_{2}$ are claimed to be completely independent. This statement is well justified in equilibrium but is quite doubtful in the presence of flow [16]. Indeed, a strong, or even moderate, flow forces the chains to align along the flow lines. Apparently, this alignment creates correlations in the orientation of 
different segments. Moreover, the DE model itself predicts catastrophic alignment of polymer chains in a flow faster than $1 / \tau_{\mathrm{d}}$ - e.g., the extinction angle predicted becomes very small in strong flows. In view of such inconsistency, there is a need for a theory in which the assumption about the absence of any correlations is not made or can be checked afterwards. It is the primary goal of this section to develop a microscopic approach generalizing the DE formalism which is free of the speculations concerning independence in the orientation of chain segments in a flow.

\section{A. Generalization of the Doi-Edwards formalism}

To generalize the DE approach, we introduce a hierarchy of distribution functions $F_{n}\left(\boldsymbol{u}_{1}, s_{1}, \ldots, \boldsymbol{u}_{n}, s_{n} ; t\right), n=1,2, \ldots$, each giving the probability that an individual chain, observed at moment $t$, has tangent vectors $\boldsymbol{u}_{i}$ at positions $s_{i}, i$ $=1, \ldots, n$. Apparently, an identity analogous to Eq. (4) holds,

$$
F_{n}\left(\boldsymbol{u}_{1}, s_{1}, \ldots, \boldsymbol{u}_{n}, s_{n} ; t\right)=\left\langle\prod_{i=1}^{n} \delta\left(\boldsymbol{u}_{i}-\hat{\boldsymbol{u}}\left(s_{i}, t\right)\right)\right\rangle,
$$

and allows one to derive a deterministic equation for $F_{n}$ based on the stochastic equation (1). Note that the "onepoint" function $F_{1}(\boldsymbol{u}, s ; t)$ corresponds to the DE probability distribution function introduced in Eq. (4).

To proceed, we consider Eq. (5) at the moment $t+\Delta t$, use $\hat{\boldsymbol{u}}(s, t+\Delta t)$ from Eq. (1), and perform an expansion up to linear terms in $\Delta t$. This gives

$$
\begin{aligned}
F_{n}\left(\boldsymbol{u}_{1}, s_{1}, \ldots, \boldsymbol{u}_{n}, s_{n} ; t+\Delta t\right)= & \left\langle\prod_{i=1}^{n} \delta\left(\boldsymbol{u}_{i}-\hat{\boldsymbol{u}}\left(s_{i}+\Delta \zeta, t\right)\right)\right\rangle \\
& -\Delta t \sum_{i=1}^{n}\left(P_{i}+Q_{i}\right) .
\end{aligned}
$$

Here we used the following notations:

$$
\begin{aligned}
P_{i} & =\left\langle\left\{\prod_{j=1, j \neq i}^{n} \delta\left(\boldsymbol{u}_{j}-\hat{\boldsymbol{u}}\left(s_{j}\right)\right)\right\} \boldsymbol{K}: \hat{\boldsymbol{u}}\left(s_{i}\right) \frac{\partial}{\partial \boldsymbol{u}_{i}} \delta\left(\boldsymbol{u}_{i}-\hat{\boldsymbol{u}}\left(s_{i}\right)\right)\right\rangle \\
& =\frac{\partial}{\partial \boldsymbol{u}_{i}} \cdot\left\langle\boldsymbol{K} \cdot \hat{\boldsymbol{u}}\left(s_{i}\right) \prod_{j=1}^{n} \delta\left(\boldsymbol{u}_{j}-\hat{\boldsymbol{u}}\left(s_{j}\right)\right)\right\rangle \\
& =\frac{\partial}{\partial \boldsymbol{u}_{i}} \cdot\left[\boldsymbol{K} \cdot \boldsymbol{u}_{i} F_{n}\left(\boldsymbol{u}_{1}, s_{1}, \ldots, \boldsymbol{u}_{n}, s_{n} ; t\right)\right]
\end{aligned}
$$

and

$$
\begin{aligned}
Q_{i}= & \left\langle\left\{\prod_{j=1, j \neq i}^{n} \delta\left(\boldsymbol{u}_{j}-\hat{\boldsymbol{u}}\left(s_{j}\right)\right)\right\} \frac{\partial}{\partial s_{i}}\left(\hat{\boldsymbol{u}}\left(s_{i}\right) \int_{0}^{s_{i}} \mathrm{~d} x \hat{\xi}(x)\right)\right. \\
& \left.\times \frac{\partial}{\partial \boldsymbol{u}_{i}} \delta\left(\boldsymbol{u}_{i}-\hat{\boldsymbol{u}}\left(s_{i}\right)\right)\right\rangle \\
= & -\left(\boldsymbol{K}: \boldsymbol{u}_{i} \boldsymbol{u}_{i}\right) F_{n}-\frac{\partial}{\partial \boldsymbol{u}_{i}} \cdot\left[\boldsymbol{u}_{i}\left(\boldsymbol{K}: \boldsymbol{u}_{i} \boldsymbol{u}_{i}\right) F_{n}\right] \\
& -\frac{\partial}{\partial s_{i}}\left\langle\left\{\prod_{j=1}^{n} \delta\left(\boldsymbol{u}_{j}-\hat{\boldsymbol{u}}\left(s_{j}\right)\right)\right\} \int_{0}^{s_{i}} \mathrm{~d} x \hat{\xi}(x)\right\rangle,
\end{aligned}
$$

where, for the sake of shortness, the time argument of $\hat{\boldsymbol{u}}(s, t)$ is omitted. Finally, the first (reptation) term on the RHS of Eq. (6) yields

$$
\left\langle\prod_{i=1}^{n} \delta\left(\boldsymbol{u}_{i}-\hat{\boldsymbol{u}}\left(s_{i}+\Delta \zeta, t\right)\right)\right\rangle=F_{n}+\Delta t D_{\mathrm{c}}\left(\sum_{i=1}^{n} \frac{\partial}{\partial s_{i}}\right)^{2} F_{n},
$$

where an expansion in $\Delta \zeta$ has been performed and the identity $\left\langle\Delta \zeta^{2}\right\rangle=2 D_{\mathrm{c}} \Delta t$ has been used.

In essence, the above derivation follows the lines of the original one by [2] although the algebra is somewhat more involved. The last term in Eq. (8) can be written in a more convenient way. First, let us recall that $\hat{\xi}(x, t)=-\boldsymbol{K}: \hat{\boldsymbol{u}}(x, t) \hat{\boldsymbol{u}}(x, t)$. Then, this term takes the form

$$
\begin{aligned}
-\sum_{i=1}^{n} & \frac{\partial}{\partial s_{i}}\left\langle\left\{\prod_{j=1}^{n} \delta\left(\boldsymbol{u}_{j}-\hat{\boldsymbol{u}}\left(s_{j}\right)\right)\right\} \int_{0}^{s_{i}} \mathrm{~d} x \boldsymbol{K}: \hat{\boldsymbol{u}}(x) \hat{\boldsymbol{u}}(x)\right\rangle \\
= & -\sum_{i=1}^{n} \frac{\partial}{\partial s_{i}} \int_{0}^{s_{i}} \mathrm{~d} x \int \mathrm{d} \boldsymbol{v}\langle\delta(\boldsymbol{v}-\hat{\boldsymbol{u}}(x)) \\
& \left.\times\left\{\prod_{j=1}^{n} \delta\left(\boldsymbol{u}_{j}-\hat{\boldsymbol{u}}\left(s_{j}\right)\right)\right\} \boldsymbol{K}: \boldsymbol{v} \boldsymbol{v}\right\rangle \\
= & -\sum_{i=1}^{n} \frac{\partial}{\partial s_{i}} \int_{0}^{s_{i}} \mathrm{~d} x \int \mathrm{d} \boldsymbol{v} F_{n+1}\left(\boldsymbol{u}_{1}, s_{1}, \ldots, \boldsymbol{u}_{n}, s_{n}, \boldsymbol{v}, x ; t\right)
\end{aligned}
$$

$\times K: v \boldsymbol{v}$

Hence, the resulting equation for the probability distribution function is given by

$$
\begin{aligned}
\frac{\partial F_{n}}{\partial t}= & D_{\mathrm{c}}\left(\sum_{i=1}^{n} \frac{\partial}{\partial s_{i}}\right)^{2} F_{n}+\sum_{i=1}^{n}\left(\boldsymbol{K}: \boldsymbol{u}_{i} \boldsymbol{u}_{i}\right) F_{n} \\
& +\sum_{i=1}^{n} \frac{\partial}{\partial \boldsymbol{u}_{i}} \cdot\left[\boldsymbol{u}_{i}\left(\boldsymbol{K}: \boldsymbol{u}_{i} \boldsymbol{u}_{i}\right) F_{n}-\boldsymbol{K} \cdot \boldsymbol{u}_{i} F_{n}\right] \\
& -\sum_{i=1}^{n} \frac{\partial}{\partial s_{i}} \int_{0}^{s_{i}} \mathrm{~d} x \int \mathrm{d} \boldsymbol{v}(\boldsymbol{K}: \boldsymbol{v} \boldsymbol{v}) F_{n+1}\left(\boldsymbol{u}_{1}, s_{1}, \ldots, \boldsymbol{u}_{n}, s_{n}, \boldsymbol{v}, x ; t\right),
\end{aligned}
$$

where the arguments of $F_{n}\left(\boldsymbol{u}_{1}, s_{1}, \ldots, \boldsymbol{u}_{n}, s_{n} ; t\right)$ are omitted for shortness.

The above-derived equation for $F_{n}$ is a deterministic equation. In its derivation no additional assumption has been made, so the result (11) is exact in the framework of Eq. (1). An important feature of Eq. (11) is that the evaluation of the $n$-point probability distribution function requires knowledge of the higher-order function $F_{n+1}$ : this situation is typical for the systems involving many-particle interactions and is encountered, e.g., in the statistical theory of simple fluids [Bogoliubov-Born-Green-Kirkwood-Yvon (BBGKY) equations; see [18]]. As will be shown further on, the DE equation for the one-point distribution function $F_{1}(\boldsymbol{u}, s ; t)$ follows from Eq. (11) as a result of a certain approximation for $F_{2}$. 
The molecular relaxation processes behind the set (11) are the same as for Eq. (1): they include reptation and retraction. As discussed in the Introduction, this picture still lacks an important ingredient responsible for the relaxation of polymer chains in relatively fast flows with rates larger than $1 / \tau_{\mathrm{d}}$-convective constraint release.

\section{B. Constraint release}

Up to this point we assumed a chain to relax in a fixed environment and neglected all the changes the tube undergoes due to the motion of the polymer chains surrounding the test chain. A consequence of such a simplification is that only longitudinal motion of a chain in its tube has been taken into account. However, the entanglement constraints building up the tube disappear and reappear in time, resulting in socalled constraint release. A distinctive feature of this relaxation mechanism is that it allows for a lateral motion of the test chain.

In slow flow, the $\mathrm{CR}$ is relatively weak-entanglements are released mainly due to reptation of the chains constituting the tube-i.e., with the frequency $W_{\mathrm{TCR}}$ of the order of $1 / \tau_{\mathrm{d}}$. This process is generally called thermal constraint release. In somewhat faster flows, typically with rates beyond $1 / \tau_{\mathrm{d}}$, another type of $\mathrm{CR}$ - convective constraint releasecomes into play. CCR has its origin in the retraction of the surrounding chains and, as far as the retraction rate is proportional to the flow rate, the frequency of CCR events increases for stronger flows. This makes CCR a major relaxation mechanism in the nonlinear flow regime.
There have been several attempts to incorporate (C)CR into a constitutive model [8-15]. Here we follow the approach by Likhtman et al. [12], who treated a CR event as a random hop of a tube segment. Each hop allows the tube segment to move a distance of the order of tube diameter $a$. Because the frequency $W$ of CR events is the same along the whole tube, this motion can be described as a Rouse-like process. Over a small interval of time $\Delta t$, it leads to a change in the position of the segment $s$ given by [13]

$$
\left.\Delta \hat{\boldsymbol{R}}(s, t)\right|_{\mathrm{CR}}=\Delta t\left[\frac{3}{2} W a^{2} \frac{\partial^{2} \hat{\boldsymbol{R}}(s, t)}{\partial s^{2}}+\hat{\boldsymbol{g}}(s, t)\right],
$$

where $\hat{\boldsymbol{g}}$ is a zero-mean Gaussian force driving the system towards equilibrium, $\quad\left\langle\hat{g}_{\alpha}\left(s_{1}, t_{1}\right) \hat{g}_{\beta}\left(s_{2}, t_{2}\right)\right\rangle=W a^{3} \delta\left(s_{1}\right.$ $\left.-s_{2}\right) \delta_{\alpha \beta} \delta\left(t_{1}-t_{2}\right)$. In our formalism, however, we need to rewrite Eq. (12) in terms of $\hat{\boldsymbol{u}}(s, t)$. Then, a "random torque" $\hat{\boldsymbol{m}}(s, t)$, which is proportional to the difference between the forces that act "at the ends" of the segment $s$, should be used instead of the random force $\hat{\boldsymbol{g}}$ :

$$
\left.\Delta \hat{\boldsymbol{u}}(s, t)\right|_{\mathrm{CR}}=\Delta t\left[\frac{3}{2} W a^{2} \frac{\partial^{2} \hat{\boldsymbol{u}}(s, t)}{\partial s^{2}}+\hat{\boldsymbol{m}}(s, t)\right] .
$$

Here $\hat{\boldsymbol{m}}$, being a difference of two Gaussian forces, is a Gaussian noise with $\left\langle\hat{m}_{\alpha}\left(s_{1}, t_{1}\right) \hat{m}_{\beta}\left(s_{2}, t_{2}\right)\right\rangle=W a \delta\left(s_{1}\right.$ $\left.-s_{2}\right) \delta_{\alpha \beta} \delta\left(t_{1}-t_{2}\right)$. This contribution (13) should be added to Eq. (1). Following the same procedure as in Sec. II A, one obtains for the correction in Eq. (11) from the first term in Eq. (13),

$$
\begin{aligned}
-\frac{3}{2} W a^{2} \sum_{i=1}^{n} \frac{\partial}{\partial \boldsymbol{u}_{i}} \cdot\left\langle\frac{\partial^{2} \hat{\boldsymbol{u}}\left(s_{i}\right)}{\partial s_{i}^{2}} \prod_{j=1}^{n} \delta\left(\boldsymbol{u}_{j}-\hat{\boldsymbol{u}}\left(s_{j}\right)\right)\right\rangle & =-\frac{3}{2} W a^{2} \sum_{i=1}^{n} \frac{\partial}{\partial \boldsymbol{u}_{i}} \cdot\left[\frac{\partial^{2}}{\partial \widetilde{s}^{2}}\left\langle\hat{\boldsymbol{u}}(\widetilde{s}) \prod_{j=1}^{n} \delta\left(\boldsymbol{u}_{j}-\hat{\boldsymbol{u}}\left(s_{j}\right)\right)\right\rangle\right]_{\tilde{s}=s_{i}} \\
& =-\frac{3}{2} W a^{2} \sum_{i=1}^{n} \frac{\partial}{\partial \boldsymbol{u}_{i}} \cdot\left[\frac{\partial^{2}}{\partial \widetilde{s}^{2}} \int \mathrm{d} \boldsymbol{v} \boldsymbol{v} F_{n+1}\left(\boldsymbol{u}_{1}, s_{1}, \ldots, \boldsymbol{u}_{n}, s_{n}, \boldsymbol{v}, \tilde{s} ; t\right)\right]_{\tilde{s}=s_{i}}
\end{aligned}
$$

The other term, originating from the random force in Eq. (13), reflects the equilibrium properties of the system and, thus, is determined by the equilibrium distribution function $F_{n}^{(\mathrm{eq})}$ only ("eq" stands for equilibrium-i.e., in the absence of flow). Under equilibrium conditions both terms must exactly cancel each other which leads to the conclusion that the CR contribution to Eq. (11) has the following explicit form [16]:

$$
\begin{aligned}
\cdots & -\frac{3}{2} W a^{2} \sum_{i=1}^{n} \frac{\partial}{\partial \boldsymbol{u}_{i}} \cdot\left[\frac { \partial ^ { 2 } } { \partial \widetilde { s } ^ { 2 } } \int \boldsymbol { v } \boldsymbol { v } \left(F_{n+1}(\ldots, \boldsymbol{v}, \widetilde{s} ; t)\right.\right. \\
& \left.\left.-F_{n+1}^{(\mathrm{eq})}(\ldots, \boldsymbol{v}, \widetilde{s})\right)\right]_{\widetilde{s}=s_{i}} .
\end{aligned}
$$

However, the Rouse motion does not preserve the constant primitive path length. To retain its length during random hops, the chain has to retract in the tube faster or slower than prescribed in the DE theory where $\hat{\xi}=-\boldsymbol{K}: \hat{\boldsymbol{u}} \hat{\boldsymbol{u}}$. Therefore, we include this effect in the retraction rate $\hat{\xi}$ and write

$$
\hat{\xi}(s, t)=-\boldsymbol{K}: \hat{\boldsymbol{u}}(s, t) \hat{\boldsymbol{u}}(s, t)+\xi_{\mathrm{CR}}(s, t),
$$

where $\xi_{\mathrm{CR}}(s, t)$ describes retraction due to hopping of the chain. Note the absence of a caret upon $\xi_{\mathrm{CR}}$-we assume it to be a universal function for all the chains. The modified $\hat{\xi}$, Eq. (16), should be used in Eq. (1) as well. It leads to an additional contribution to Eq. (11): 


$$
\cdots+\sum_{i=1}^{n}\left[\left(\int_{0}^{s_{i}} \mathrm{~d} x \xi_{\mathrm{CR}}(x)\right) \frac{\partial F_{n}}{\partial s_{i}}-\xi_{\mathrm{CR}}\left(s_{i}\right) \frac{\partial}{\partial \boldsymbol{u}_{i}} \cdot\left(\boldsymbol{u}_{i} F_{n}\right)\right] .
$$

The function $\xi_{\mathrm{CR}}(s, t)$ is yet unknown-it will be calculated from the "constant-segment-length" condition $\left\langle\hat{\boldsymbol{u}}(s, t)^{2}\right\rangle \equiv 1$ further on. Note that in contrast with Milner et al. [13], we do not assume the retraction rate $\hat{\xi}(s, t)$ to be independent of the position along the chain $s$. Instead, an analytical expression for it will be derived in Sec. III.

Finally, the CR rate $W$ is determined self-consistently. As pointed out above, the convective constraint release rate $W_{\mathrm{CCR}}$ is related to the retraction rate $\hat{\xi}$. Indeed, every time a chain retracts over a distance equal to tube diameter $a$, an entanglement with another chain is destroyed. This leads to

$$
W_{\mathrm{CCR}}=-\frac{2 C_{W}}{L} \int_{0}^{L / 2} \mathrm{~d} x\langle\hat{\xi}(x, t)\rangle
$$

where, following Milner et al. [13], we have introduced a numerical constant $C_{W}$. As explained elsewhere [12-14], this phenomenological coefficient can be interpreted as the reciprocal number of chains participating (on average) in one entanglement. Thus, one expects $C_{W}<1$. A concrete value of the constant can be fixed based on the steady shear flow data in the nonlinear regime $[13,14]$.

Independently of convection, thermal CR (TCR) takes place due to reptation. The TCR rate $W_{\mathrm{TCR}}$ is derived from the same argument as Eq. (18) and reads

$$
W_{\mathrm{TCR}}=C_{W} \frac{12}{\pi^{2} \tau_{\mathrm{d}}} .
$$

Hence, the total CR rate $W$ present in Eq. (15) takes the form

$$
W=C_{W}\left[\frac{12}{\pi^{2} \tau_{\mathrm{d}}}-\frac{2}{L} \int_{0}^{L / 2} \mathrm{~d} x\langle\hat{\xi}(x, t)\rangle\right] .
$$

\section{Contour length fluctuations}

Besides retraction, reptation, and constraint release, included in Eqs. (11), (15), and (18), yet another relaxation mechanism is believed to be important. Its essence is in the fast relaxation of the end portions of the primitive path: typically, about $\sqrt{Z}$ segments, where $Z=L / a$ is the total number of entanglements per chain, can relax the stress (faster than by reptation) due to fluctuations of the chain length [2]. This process, called relaxation by contour length fluctuations (CLF), is especially important for the linear relaxation of relatively short polymers, with $Z<100$. One of the consequences of CLF is the phenomenological 3.4-power law for the zero-shear viscosity $[5,6]$ observed in experiment, whereas power 3 is predicted by the DE theory.

A rigorous incorporation of CLF into constitutive modeling is quite a formidable problem. However, an approximate treatment $[11,13,15]$ is possible if a term of the form $-\left[S(s)-S^{(\mathrm{eq})}(s)\right] / \tau_{\mathrm{CLF}}(s)$ is added to the relaxation equation for a quantity $S$ (which can be, e.g., an orientation tensor of some other microscopic characteristic) with $\tau_{\mathrm{CLF}}(s)$ being the time needed to relax an "arm" of $L / 2-|s|$ segments by CLF. This microscopic time can be borrowed from more detailed theories of linear relaxation in star-polymer melts. Here we use the result by Milner and McLeish [19],

$$
\tau_{\mathrm{CLF}}(s)=\frac{\tau_{\text {early }}\left(1-\frac{2|s|}{L}\right) \exp \left[\frac{3 Z}{4}\left(1-\frac{2|s|}{L}\right)^{2}\right]}{1+\exp \left[\frac{3 Z}{4}\left(1-\frac{2|s|}{L}\right)^{2}\right] \tau_{\text {early }}\left(1-\frac{2|s|}{L}\right) / \tau_{\text {late }}\left(1-\frac{2|s|}{L}\right)},
$$

where

$$
\begin{gathered}
\tau_{\text {early }}(x)=\frac{3 \pi^{3}}{16} Z \tau_{\mathrm{d}}\left(\frac{x}{4}\right)^{4}, \\
\tau_{\text {late }}(x)=\frac{\tau_{\mathrm{d}}}{12 Z} \mathrm{e}^{3 Z x^{2} / 4} .
\end{gathered}
$$

One also has to notice that the $n$-point distribution function $F_{n}\left(\boldsymbol{u}_{1}, s_{1}, \ldots, \boldsymbol{u}_{n}, s_{n} ; t\right)$ "has relaxed" when the "fastest"-i.e., the closest to one of the ends-segment $\max _{i=1, \ldots, n}\left(\left|s_{i}\right|\right)$ has relaxed. This furnishes for an additional term in Eq. (11) due to $\mathrm{CLF}$ :

$$
\cdots-\frac{F_{n}-F_{n}^{(\mathrm{eq})}}{\tau_{\mathrm{CLF}}\left(\max _{i=1, \ldots, n}\left|s_{i}\right|\right)} .
$$

Apart from a faster relaxation of the chain ends, the presence of CLF also influences the thermal constraint release rate. Indeed, an effective disengagement time can be introduced [2]:

$$
\tau_{\mathrm{d}}^{(\mathrm{eff})}=\tau_{\mathrm{d}}\left(1-\xi_{d}\right)^{2},
$$

taking into account that a $\xi_{d}$ fraction of the primitive path relaxes faster than it would by reptation and, effectively, the tube from which the chain should escape has the length of $L\left(1-\xi_{d}\right)$. The portion $\xi_{d}$ is obtained from an equality $\tau_{\mathrm{d}}(1$ $\left.-\xi_{d}\right)=\tau_{\text {early }}\left(\xi_{d}\right)$ (see [6] for more details). The latter equation for $\xi_{d}$ can be solved numerically. However, for practical pur- 
poses, its approximate solution in the form of a perturbative series with $1 / Z$ as a small parameter,

$$
\xi_{d}=\frac{4}{\left(3 \pi^{3} Z\right)^{1 / 4}}\left[1-\frac{2}{\left(3 \pi^{3} Z\right)^{1 / 4}}+\frac{2}{\left(3 \pi^{3} Z\right)^{1 / 2}}+\cdots\right],
$$

is indistinguishable from the exact numerical one even for $Z=10$. To consider the effect of the tube shortening on the TCR rate, we substitute $\tau_{\mathrm{d}}^{\text {eff }}$, Eq. (25), instead of $\tau_{\mathrm{d}}$ in Eq. (19) - this enhances the TCR rate, especially for relatively short chains, as expected. Then, the final expression for the constraint release rate reads

$$
W=C_{W}\left[\frac{12}{\pi^{2} \tau_{\mathrm{d}}\left(1-\xi_{d}\right)^{2}}-\frac{2}{L} \int_{0}^{L / 2} \mathrm{~d} x\langle\hat{\xi}(x, t)\rangle\right],
$$

where $\xi_{d}$ is given by Eq. (26).

To summarize, the set of equations (11) with additional terms (15), (17), and (24) forms the basis for studying the dynamics of linear entangled polymers. They describe relaxation due to reptation, retraction, constraint release, and contour length fluctuations and take into account correlations along the chain which may become important due to the possible orientation of a primitive path under flow conditions. To complete the system, the CR rate $W$ is calculated self-consistently in Eq. (27). Moreover, the set (11) should be solved under an additional condition of a constant segmental length $\left\langle\hat{\boldsymbol{u}}(s, t)^{2}\right\rangle=\int \mathrm{d} \boldsymbol{u} \boldsymbol{u}^{2} F_{1}(\boldsymbol{u}, s ; t) \equiv 1$, from which the yet unknown function $\xi_{\mathrm{CR}}(s, t)$ can be obtained.

\section{RESULTS AND DISCUSSION}

The set of equations for the $F_{n}$ 's, derived in the previous section, is quite general. However, its complex structure makes it hardly tractable. Indeed, to solve an equation for $F_{n}$, one requires knowledge of $F_{n+1}$ and so forth. Of course, a physical cutoff should be applied at $n=Z$, but as far as one is typically interested in $Z \gtrsim 10$, it does not simplify the task. A somewhat similar problem, involving a chain of distribution functions, is encountered in the statistical physics of fluids where the famous BBGKY set of equations for the density distribution functions is quite well studied [18]. To solve it, one generally introduces some approximation based on a physical assumption that the $(n+1)$ th-order function can be expressed in terms of the previous ones. Such an approximation, called a closure, allows one to derive a much simpler set of equations, restricted to a small number (typically, one or two) of distribution functions: e.g., the well-known Kirkwood's superposition approximation generates an equation for the pair correlation function.

In what follows, we show that an analogous approach can be employed in our situation as well. Simple closure approximations for the higher-order distribution functions will be demonstrated to yield some existing models and shed some light on the connection between them. In some cases, a more detailed consideration allows one to avoid the unnecessary approximations often encountered in the literature.

\section{A. "One-point" closure approximation and the DE theory}

As has been pointed out already, one of the assumptions in the DE theory is the independence of the orientation of different segments along the primitive path. In the language of the probability distribution functions, it means that a onepoint function $F_{1}(\boldsymbol{u}, s ; t)$ is enough for a complete description of the system and all the higher-order functions can be expressed as products of one-point contributions. In particular, $F_{2}$ is written as $F_{2}\left(\boldsymbol{u}_{1}, s_{1}, \boldsymbol{u}_{2}, s_{2} ; t\right)$ $=F_{1}\left(\boldsymbol{u}_{1}, s_{1} ; t\right) F_{1}\left(\boldsymbol{u}_{2}, s_{2} ; t\right)$. Using this closure approximation, the last term in Eq. (11) for $n=1$ reduces to

$$
\begin{aligned}
& -\frac{\partial}{\partial s} \int_{0}^{s} \mathrm{~d} x \int \mathrm{d} \boldsymbol{v}(\boldsymbol{K}: \boldsymbol{v} \boldsymbol{v}) F_{1}(\boldsymbol{u}, s ; t) F_{1}(\boldsymbol{v}, x ; t) \\
& \quad=-\frac{\partial}{\partial s}\left[F_{1}(\boldsymbol{u}, s ; t) \int_{0}^{s} \mathrm{~d} x\langle\boldsymbol{K}: \hat{\boldsymbol{u}}(x, t) \hat{\boldsymbol{u}}(x, t)\rangle\right]
\end{aligned}
$$

and Eq. (11) gives

$$
\begin{aligned}
\frac{\partial F_{1}}{\partial t}= & {\left[D_{\mathrm{c}} \frac{\partial^{2}}{\partial s^{2}}-\int_{0}^{s} \mathrm{~d} x\langle\boldsymbol{K}: \hat{\boldsymbol{u}}(x) \hat{\boldsymbol{u}}(x)\rangle \frac{\partial}{\partial s}\right] F_{1} } \\
& +\frac{\partial}{\partial \boldsymbol{u}} \cdot\left[\boldsymbol{u}(\boldsymbol{K}: \boldsymbol{u u}) F_{1}-\boldsymbol{K} \cdot \boldsymbol{u} F_{1}\right] \\
& +[(\boldsymbol{K}: \boldsymbol{u u})-\langle\boldsymbol{K}: \hat{\boldsymbol{u}}(s) \hat{\boldsymbol{u}}(s)\rangle] F_{1},
\end{aligned}
$$

which exactly coincides with the DE equation without independent alignment [2]. Note that we did not include the CR and CLF terms because they were not present in the original treatment by Doi and Edwards [2]. As will be shown further on, constraint release included in the "one-point" formalism leads to a constitutive equation similar to the one obtained by Ianniruberto and Marrucci [10].

\section{B. "Two-point" closure approximation}

To go beyond the simple one-point approximation, one has to consider closures involving higher-order distribution functions-e.g., $F_{2}$. The two-point distribution function not only contains information about the orientation of a primitive path segment but also allows one to estimate how important the effect of the flow-induced orientation correlations is. Traditionally, predicting rheological properties, one would focus on the orientation tensor $\boldsymbol{S}(s, t) \equiv\langle\hat{\boldsymbol{u}}(s, t) \hat{\boldsymbol{u}}(s, t)\rangle$. However, knowledge of the two-point distribution function $F_{2}$ allows for a more detailed description based on a more general twopoint correlator $\left[\boldsymbol{G}\left(s_{1}, s_{2} ; t\right)\right] \equiv\left\langle\hat{\boldsymbol{u}}\left(s_{1}, t\right) \hat{\boldsymbol{u}}\left(s_{2}, t\right)\right\rangle$. Computationally, it is much simpler to work with $\boldsymbol{G}$ than with $F_{2}$ itself because $\boldsymbol{G}$ is a function of scalar arguments. Therefore, in the rest of this section, we shall mainly focus on an equation of motion for this correlation tensor.

An evolution equation for $\boldsymbol{G}$ can be readily obtained from the one for $F_{2}$ using the definition $\boldsymbol{G}\left(s_{1}, s_{2} ; t\right)$ $=\int \mathrm{d} \boldsymbol{u}_{1} \int \mathrm{d} \boldsymbol{u}_{2} \boldsymbol{u}_{1} \boldsymbol{u}_{2} F_{2}\left(\boldsymbol{u}_{1}, s_{1}, \boldsymbol{u}_{2}, s_{2} ; t\right)$. Omitting the algebra, we just highlight the most important steps of the derivation. A typical term from the CR contribution (15), taken at $n=2$, yields 


$$
\begin{aligned}
& -\frac{3 W a^{2}}{2} \int \mathrm{d} \boldsymbol{u}_{1} \int \mathrm{d} \boldsymbol{u}_{2} \boldsymbol{u}_{1} \boldsymbol{u}_{2} \frac{\partial}{\partial \boldsymbol{u}_{1}} \cdot\left[\frac{\partial^{2}}{\partial \widetilde{s}^{2}} \int \mathrm{d} \boldsymbol{v} \boldsymbol{v} F_{3}\left(\boldsymbol{u}_{1}, s_{1}, \boldsymbol{u}_{2}, s_{2}, \boldsymbol{v}, \widetilde{s}\right)\right]_{\tilde{s}=s_{1}} \\
& =\frac{3 W a^{2}}{2}\left[\frac{\partial^{2}}{\partial \widetilde{s}^{2}} \int \mathrm{d} \boldsymbol{u}_{1} \int \mathrm{d} \boldsymbol{u}_{2} \int \mathrm{d} \boldsymbol{v} F_{3} \boldsymbol{v} \cdot \frac{\partial}{\partial \boldsymbol{u}_{1}} \cdot \boldsymbol{u}_{1} \boldsymbol{u}_{2}\right]_{\widetilde{s}=s_{1}}=\frac{3 W a^{2}}{2} \frac{\partial^{2}}{\partial s_{1}^{2}} \boldsymbol{G}\left(s_{1}, s_{2} ; t\right),
\end{aligned}
$$

where additionally the identity $F_{2}=\int \mathrm{d} \boldsymbol{u}_{3} F_{3}$ has been used. Next, the last term in Eq. (11), which in the case $n=2$ contains $F_{3}$, needs some special attention: a closure approximation has to be introduced to express $F_{3}$ in terms of $F_{1}$ and $F_{2}$. The simplest approximate reduction, fulfilling the symmetry condition and the above-mentioned identity, reads as

$$
\begin{aligned}
F_{3}\left(A_{1}, A_{2}, A_{3}\right)= & F_{1}\left(A_{1}\right) F_{1}\left(A_{2}\right) F_{1}\left(A_{3}\right)+\sum_{\{(i, j, k)\}}\left[F_{2}\left(A_{i}, A_{j}\right)\right. \\
& \left.-F_{1}\left(A_{i}\right) F_{1}\left(A_{j}\right)\right] F_{1}\left(A_{k}\right),
\end{aligned}
$$

where $A_{i}$ denotes a pair $\boldsymbol{u}_{i}, s_{i}$ and the summation is conducted over a cyclic permutation of the indices $\{(i, j, k)\}$ $\equiv\{(1,2,3),(2,3,1),(3,1,2)\}$. The physical meaning of Eq. (30) is clear: it implies that all three-point correlations can be approximated as a combination of one- and two-point terms and all irreducible three-point contributions are neglected. Such an approximation (30) allows one to obtain a closed equation for $F_{2}$ or, equivalently, for $\boldsymbol{G}$, which after some algebra leads to

$$
\begin{aligned}
\frac{\partial \boldsymbol{G}}{\partial t}= & D_{\mathrm{c}}\left(\frac{\partial}{\partial s_{1}}+\frac{\partial}{\partial s_{2}}\right)^{2} \boldsymbol{G}+\boldsymbol{K} \cdot \boldsymbol{G}+\boldsymbol{G} \cdot \boldsymbol{K}^{T}-\left[\boldsymbol{K}: \boldsymbol{G}\left(s_{1}, s_{1} ; t\right)\right. \\
& \left.+\boldsymbol{K}: \boldsymbol{G}\left(s_{2}, s_{2} ; t\right)\right] \boldsymbol{G}+\sum_{i=1,2}\left[\xi_{\mathrm{CR}}\left(s_{i}\right) \boldsymbol{G}\right. \\
& \left.-\left(\int_{0}^{s_{i}} \mathrm{~d} x\left[\boldsymbol{K}: \boldsymbol{G}(x, x ; t)-\xi_{\mathrm{CR}}(x)\right]\right) \frac{\partial \boldsymbol{G}}{\partial s_{i}}\right]+\frac{3 W a^{2}}{2}\left(\frac{\partial^{2}}{\partial s_{1}^{2}}\right. \\
& \left.+\frac{\partial^{2}}{\partial s_{2}^{2}}\right)\left(\boldsymbol{G}-\boldsymbol{G}^{(\mathrm{eq})}\right)-\frac{\boldsymbol{G}-\boldsymbol{G}^{(\mathrm{eq})}}{\tau_{\mathrm{CLF}}\left(\max _{i=1, \ldots, n}\left|s_{i}\right|\right)}
\end{aligned}
$$

where the arguments of $\boldsymbol{G}$ are $\left(s_{1}, s_{2} ; t\right)$ if not indicated otherwise. Equation (31) is strikingly similar to the one by Milner et al. [13]. However, in the present approach, it results from a well-defined approximation to a more general formalism which allows one to avoid unnecessary simplifications: e.g., we did not assume the retraction rate to be constant and explicitly separated its component $\xi_{\mathrm{CR}}$ due to CR.

The equation of motion (31) is a nonlinear partial integrodifferential equation for $\boldsymbol{G}$. Its equilibrium solution (no flow) $\boldsymbol{G}^{(\mathrm{eq})}\left(s_{1}, s_{2}\right)$ is an isotropic tensor depending only on the distance between $s_{1}$ and $s_{2}$ :

$$
\boldsymbol{G}^{(\mathrm{eq})}\left(s_{1}, s_{2}\right)=\frac{\boldsymbol{I}}{3} \Delta\left(s_{1}-s_{2}\right),
$$

where $\Delta(x)$ is a positive bell-shaped function with maximum $\Delta_{\max }=1$ at $x_{\max }=0$ and width proportional to the correlation length at equilibrium-i.e., the segment size $a$. The specific shape of the function $\Delta$, as numerical results show, is not of a crucial importance if the conditions $\Delta(0)=1$ and $\lim _{a \rightarrow 0} \Delta(s) / a=\delta(s)$ are fulfilled. For convenience, we take

$$
\Delta(x)=\exp \left(-\pi \frac{x^{2}}{a^{2}}\right) .
$$

Also, boundary conditions on the rectangle $-L / 2 \leqslant s_{1,2}$ $\leqslant L / 2$ should complement Eq. (31). Assuming that the ends of a chain are always relaxed, we use the condition

$$
\left.\boldsymbol{G}\left(s_{1}, s_{2} ; t\right)\right|_{\text {boundary }}=\left.\boldsymbol{G}^{(\mathrm{eq})}\left(s_{1}, s_{2}\right)\right|_{\text {boundary }} \text {. }
$$

As also discussed in [13], it is impossible to reduce Eq. (31) to a more restricted characteristic than $\boldsymbol{G}$. This complexity arises because the reptative diffusion acts along the diagonal lines $s_{1}-s_{2}=$ const whereas CR acts isotropically. However, it is still useful to rewrite Eq. (31) in more "natural" coordinates

$$
v=\frac{s_{1}+s_{2}}{2}, \quad w=s_{1}-s_{2} .
$$

After some trivial algebra, the result reads

$$
\begin{aligned}
\frac{\partial \widetilde{\boldsymbol{G}}}{\partial t}= & D_{\mathrm{c}} \frac{\partial^{2} \widetilde{\boldsymbol{G}}}{\partial v^{2}}+\boldsymbol{K} \cdot \widetilde{\boldsymbol{G}}+\widetilde{\boldsymbol{G}} \cdot \boldsymbol{K}^{T}-[\boldsymbol{K}: \widetilde{\boldsymbol{G}}(v+w / 2,0 ; t)+\boldsymbol{K}: \widetilde{\boldsymbol{G}}(v \\
& -w / 2,0 ; t)] \widetilde{\boldsymbol{G}}-\left[\int_{0}^{v+w / 2} \mathrm{~d} x\left[\boldsymbol{K}: \widetilde{\boldsymbol{G}}(x, 0 ; t)-\xi_{\mathrm{CR}}(x)\right]\right] \\
& \times\left(\frac{1}{2} \frac{\partial \widetilde{\boldsymbol{G}}}{\partial v}+\frac{\partial \widetilde{\boldsymbol{G}}}{\partial w}\right)+\xi_{\mathrm{CR}}(v+w / 2) \widetilde{\boldsymbol{G}} \\
& -\left[\int_{0}^{v-w / 2} \mathrm{~d} x\left[\boldsymbol{K}: \widetilde{\boldsymbol{G}}(x, 0 ; t)-\xi_{\mathrm{CR}}(x)\right]\right]\left(\frac{1}{2} \frac{\partial \widetilde{\boldsymbol{G}}}{\partial v}-\frac{\partial \widetilde{\boldsymbol{G}}}{\partial w}\right) \\
& +\xi_{\mathrm{CR}}(v-w / 2) \widetilde{\boldsymbol{G}}+\frac{3 W a^{2}}{2}\left(\frac{1}{2} \frac{\partial^{2}}{\partial v^{2}}+2 \frac{\partial^{2}}{\partial w^{2}}\right)[\widetilde{\boldsymbol{G}}(v, w ; t) \\
& \left.-\widetilde{\boldsymbol{G}}^{(\mathrm{eq})}(w)\right]-\frac{\widetilde{\boldsymbol{G}}-\widetilde{\boldsymbol{G}}^{(\mathrm{eq})}}{\tau_{\mathrm{CLF}}},
\end{aligned}
$$

where the new tensor $\widetilde{\boldsymbol{G}}(v, w ; t)=\boldsymbol{G}\left(s_{1}, s_{2} ; t\right)$ has been introduced. The last equation demonstrates clearly that the problem without CR can be easily reduced to the one of finding the orientation tensor $\boldsymbol{S}(v, t)=\widetilde{\boldsymbol{G}}(v, 0 ; t)$ by taking $w=0$ in Eq. (36). However, the presence of the isotropic CR term complicates things and results in the following equation for $S$ : 


$$
\begin{aligned}
\frac{\partial \boldsymbol{S}}{\partial t}= & \left(D_{\mathrm{c}}+\frac{3 W a^{2}}{4}\right) \frac{\partial^{2} \boldsymbol{S}}{\partial s^{2}}+\boldsymbol{K} \cdot \boldsymbol{S}+\boldsymbol{S} \cdot \boldsymbol{K}^{T}-2(\boldsymbol{K}: \boldsymbol{S}) \boldsymbol{S}-\left[\int_{0}^{s} \mathrm{~d} x\left[\boldsymbol{K}: \boldsymbol{S}(x, t)-\xi_{\mathrm{CR}}(x)\right]\right] \frac{\partial \boldsymbol{S}}{\partial s}+2 \xi_{\mathrm{CR}}(s) \boldsymbol{S}-\frac{\boldsymbol{S}-\boldsymbol{I} / 3}{\tau_{\mathrm{CLF}}} \\
& +3 W a^{2}\left[\frac{\partial^{2}\left(\widetilde{\boldsymbol{G}}(s, w ; t)-\widetilde{\boldsymbol{G}}^{(\mathrm{eq})}(w)\right)}{\partial w^{2}}\right]_{w=0} \cdot
\end{aligned}
$$

At this point the unknown function $\xi_{\mathrm{CR}}(x, t)$ can be calculated. We recall that $\xi_{\mathrm{CR}}$ governs the additional retraction rate to compensate the stretching due to $\mathrm{CR}$ and enforce the condition $\operatorname{Tr} \boldsymbol{S}(s, t) \equiv\left\langle\hat{\boldsymbol{u}}(s, t)^{2}\right\rangle=1$. Taking the trace of Eq. (37) and using the definition of the CR rate (27), one obtains

$$
\xi_{\mathrm{CR}}(s, t)=-\frac{3 C_{W} a^{2} X(s, t)\left[6 /\left[\pi^{2} \tau_{\mathrm{d}}\left(1-\xi_{d}\right)^{2}\right]+\int_{0}^{L / 2} \mathrm{~d} x \boldsymbol{K}: \widetilde{\boldsymbol{G}}(x, 0) / L\right]}{1-3 C_{W} a^{2} \int_{0}^{L / 2} \mathrm{~d} x X(x, t) / L},
$$

where $X(s, t) \equiv\left\{\partial^{2} \operatorname{Tr}\left[\widetilde{\boldsymbol{G}}(s, w ; t)-\widetilde{\boldsymbol{G}}^{(\mathrm{eq})}(w, t)\right] / \partial w^{2}\right\}_{w=0}$. Hence, Eq. (37) allows one to derive the retraction rate due to CR in a simple explicit form and avoid some of the assumptions used in [13]. This expression for $\xi_{\mathrm{CR}}$, substituted into Eq. (36), turns it into a closed equation for the correlation tensor $\widetilde{\boldsymbol{G}}$.

\section{Limiting case of a one-point theory with $C R$}

Rigorously, it is impossible to reduce Eq. (37) to a closed equation for $\boldsymbol{S}(s ; t) \equiv\langle\hat{\boldsymbol{u}}(s, t) \hat{\boldsymbol{u}}(s, t)\rangle$. Despite this fact, a lot of theories $[8-10,15]$ (for the sake of shortness we shall call them "one-point theories" further on) succeeded in incorporating the (C)CR process in the equation of motion for $S$ : they modified the relaxation time $\tau$ by adding some convective term proportional to $\boldsymbol{K}: \boldsymbol{S}$ or, in other words, to the hopping rate $W$ :

$$
\frac{1}{\tau} \sim \frac{1}{\tau_{\mathrm{d}}}+W
$$

However, the two addends in Eq. (39) act on absolutely different scales: the reptation time is a characteristic relaxation time of the whole chain whereas $1 / W$ is the lifetime of one constraint and is local. So one could expect [20] the appearance of the large-scale relaxation time $Z^{2} / W$ instead of $1 / W$ in Eq. (39). However, this would make the CR effect negligible (in monodisperse melts, in comparison to reptation) and would not improve the predictions of the rheological behavior in nonlinear flows as compared to the original DE theory.

To summarize, although in practice Eq. (39) leads to satisfactory results (see, e.g., $[8,9,15]$ ), from the theoretical point of view it does not directly agree with the expectation raised by the molecular picture of the process. Correspondingly, it is the goal of the present section to show why a relaxation time like that of Eq. (39) can be used and gives quite accurate results.

It is clear that Eq. (37) obtained for $S$ is not closed: it still includes a derivative of the tensor $\widetilde{\boldsymbol{G}}$ in the last term. To proceed, we recall that one of the essential assumptions made in the one-point theories is that correlations decay on the length scale of $a$ even in the presence of a flow. This implies an ansatz of the form

$$
\widetilde{\boldsymbol{G}}(v, w) \simeq \boldsymbol{S}(v) \Delta(w),
$$

where $\Delta(w)$ is the function introduced earlier which describes correlations in the equilibrium state [see Eq. (32)]. In essence, an assumption like Eq. (40) implies that correlations under flow conditions are the same as in equilibrium. Using the ansatz (40) and taking the Gaussian form for $\Delta(w)$, Eq. (33), one may transform Eq. (37) into the closed form

$$
\begin{aligned}
\frac{\partial \boldsymbol{S}(s, t)}{\partial t}= & \left(D_{\mathrm{c}}+\frac{3 W a^{2}}{4}\right) \frac{\partial^{2} \boldsymbol{S}}{\partial s^{2}}-6 \pi W\left(\boldsymbol{S}-\frac{\boldsymbol{I}}{3}\right)+\boldsymbol{K} \cdot \boldsymbol{S}+\boldsymbol{S} \cdot \boldsymbol{K}^{T} \\
& -2(\boldsymbol{K}: \boldsymbol{S}) \boldsymbol{S}-\left[\int_{0}^{s} \mathrm{~d} x \boldsymbol{K}: \boldsymbol{S}(x, t)\right] \frac{\partial \boldsymbol{S}}{\partial s}-\frac{\boldsymbol{S}-\boldsymbol{I} / 3}{\tau_{\mathrm{CLF}}(s)} .
\end{aligned}
$$

Let us discuss the result (41) briefly. The influence of the CCR part that acts in "parallel" to reptation (see the first brackets on the RHS) is negligible as far as $D_{c} \gg W a^{2} / 2$. However, the remaining part originating from the $w$-coordinate contribution is important. The effective relaxation time $\tau$ calculated on the basis of Eq. (41) reads $1 / \tau$ $\simeq\left(D_{\mathrm{c}}+W a^{2} / 2\right) / L^{2}+6 \pi W \sim 1 / \tau_{\mathrm{d}}+W$ (we omitted the small term $W a^{2} / L^{2}$ ) and corresponds to the relation (39), which is taken in the one-point theories for granted. Note that the important CR term $-6 \pi W(\boldsymbol{S}-\boldsymbol{I} / 3)$ appeared not due to some one-mode approximation (cf. [21]) - $\boldsymbol{S}(s, t)$ is still a function of the position along the chain-but as a consequence of the assumption that each segment undergoes Rouse motion perpendicular to the primitive path independently of the neighboring segments. This type of motion resembles orientational diffusion experienced by each segment independently of the others [22]. Naturally, the relaxation time of such a process is of the order of the constraint removal time $1 / W$ supporting Eq. (39). In contrast, the time needed to renew the conformation by translational diffusion (due to CCR) is $Z^{2}$ times 
longer; this process acts in the same way as reptation does and leads to a negligible correction.

\section{Numerical analysis}

Equation (36) together with the initial and boundary conditions and Eqs. (27) and (38) for the constraint release rate form a closed system, which can be solved numerically. Traditionally, one would determine the model parameters, such as $\tau_{\mathrm{d}}$, etc., based on some available rheological data set and discuss the quality of the fit obtained. We however pursue a different goal. Rather than fitting the data, we would like to discuss the influence that one or another approximation has on the predictions. In this respect, the formalism presented here allows one to compare the effects the different assumptions lead to: e.g., predictions in the "one-point" limit (41) can be confronted with the ones given by a more general "two-point" approximation (36). We believe such a comparison sheds some light on the relation between the known theories [8-15] and the assumptions they are based on.

All the numerical results presented below are obtained by a real-space solution of the corresponding partial differential equation [e.g., Eq. (41) or (36)] by a finite-difference scheme [23]. For a cross-check, both explicit and Peacem-Rachford alternating direction implicit methods have been tested to give the same results. The function $\Delta(x)$ in the initial condition (32) has been chosen as in Eq. (33). Alternatively, as proposed in [14], the function

$$
\Delta(x)= \begin{cases}0, & |x|>a / 2, \\ 1, & |x| \leqslant a / 2,\end{cases}
$$

could be used-for all the results presented here it yields the same predictions.

a. Transient and steady shear flows.

Transient behavior of the system in a shear flow is similar to the one predicted by Milner, McLeish, and Likhtman (MML) [13]. The stress component $\sigma_{x y}$ shows overshoots for flows with rates higher than $1 / \tau_{\mathrm{d}}$, whereas, because no chain stretch has been included into the model, overshoots are never observed for the first normal stress difference $\sigma_{x x}$ $-\sigma_{y y}$. Integrating the equations for a startup of a simple shear for a sufficiently long time, one enters the steady-state regime. The quantities of interest here apparently include the steady-state stresses as functions of the shear rate and the extinction angle. As is well known, the Doi-Edwards theory $[2,11,24]$ fails to reproduce certain features of these curves. In particular, the predicted nonmonotonicity of $\sigma_{x y}$ as a function of $\dot{\gamma}$ is one of the edge stones of both the DE and Doi, Edwards, Marrucci, and Grizzuti (DEMG) [7] theories. Constraint release is known to remove this problem $[8,9,11-13]$.

Indeed, as seen from Fig. 2(a), for $C_{W}=0$, corresponding to the absence of CR, the shear stress reaches a maximum at approximately $\dot{\gamma}=1 / \tau_{\mathrm{d}}$, as expected. While increasing $C_{W}$, the maximum first becomes less pronounced and finally disappears at $C_{W}$ close to 0.1 . This provides a good estimate for $C_{W}$ which we shall use further on. It is also clear that CCR is mainly active in nonlinear flows with a rate larger than the
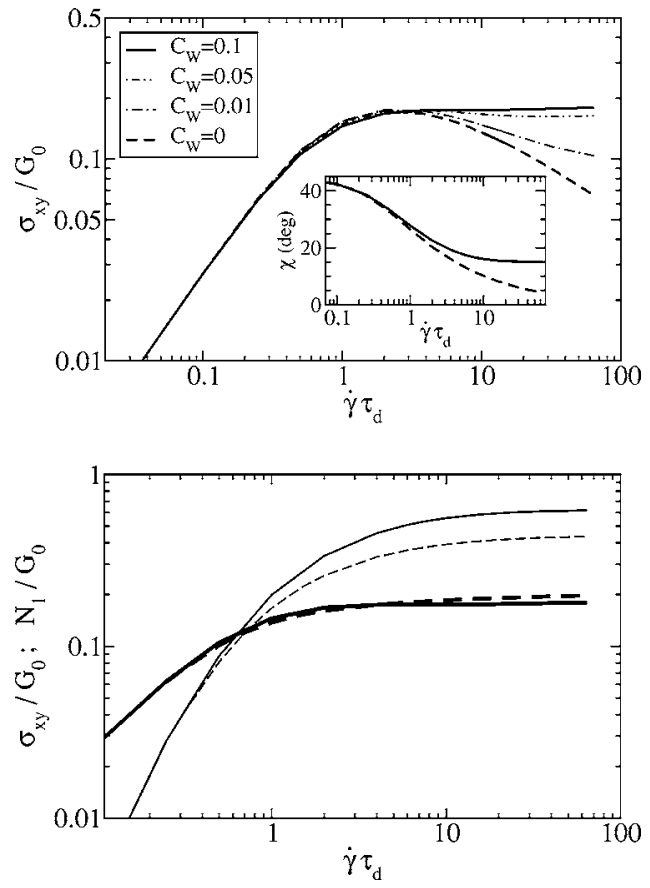

FIG. 2. (a) Steady-state shear stress $\sigma_{x y}$ vs shear rate $\dot{\gamma}$ : no CLF is included, $C_{W}=0,0.01,0.05,0.1$. For $C_{W}=0$, as expected, a maximum around $\dot{\gamma} \tau_{\mathrm{d}} \sim 1$ is observed. Inset: extinction angle $\chi$ determined via the stress-optical law $\chi=0.5 \arctan \left[2 \sigma_{x y} /\left(\sigma_{x x}-\sigma_{y y}\right)\right]$. (b) Steady-state shear stresses $\sigma_{x y}$ (thick lines) and $N_{1}$ (thin lines) vs shear rate $\dot{\gamma}$ for $C_{W}=0.1$. Solid lines correspond to Eq. (36); dashed lines represent a solution of Eq. (41) under the same conditions.

inverse reptation time: for values $\dot{\gamma} \tau_{\mathrm{d}}<1$ the curves in Fig. 2(a) are almost indistinguishable.

The more realistic behavior of the theory in the presence of constraint release is a consequence of its qualitatively accurate prediction of the extinction angle $\chi$. To compare the degree of the chain orientation with and without $\mathrm{CR}$ we plotted $\chi$ as a function of the shear rate for $C_{W}=0$ and $C_{W}=0.1$, inset in Fig. 2(a). A too rapid drop of the angle between the dominant principal axis of the stress tensor and the direction of the flow is known [11] to be one of the disadvantages of the DE theory. Figure 2(a) shows that constraint release eliminates this flaw and, for $\dot{\gamma} \tau_{\mathrm{d}}>1$, leads to a less steep curve. Moreover, it apparently approaches a limiting angle of about $15^{\circ}$, in accordance with predictions by Milner et al. [13]. Constraint release misaligns the tube and helps the flow to hold a grasp on the chain. In contrast, without CR the chain seems to be almost completely aligned with the flow and the latter loses its grip-this yields the nonmonotonic behavior of $\sigma_{x y}(\dot{\gamma})$ never observed in experiments.

It is also worthy to compare the predictions of the "twopoint" approximation (31) to the ones of the "one-point" limit (41). The predicted steady stresses $\sigma_{x y}$ are close to each other, Fig. 2(b). However, a nonvanishing systematic disagreement in values of the first normal stress $N_{1}=\sigma_{x x}-\sigma_{y y}$ clearly appears in the flows faster than $1 / \tau_{\mathrm{d}}$. Apparently, this indicates that assumption (40) approximately holds for the $x y$ components but is very poor for the $x x$ one. This is what one can indeed expect: the chain becomes preferably oriented in 


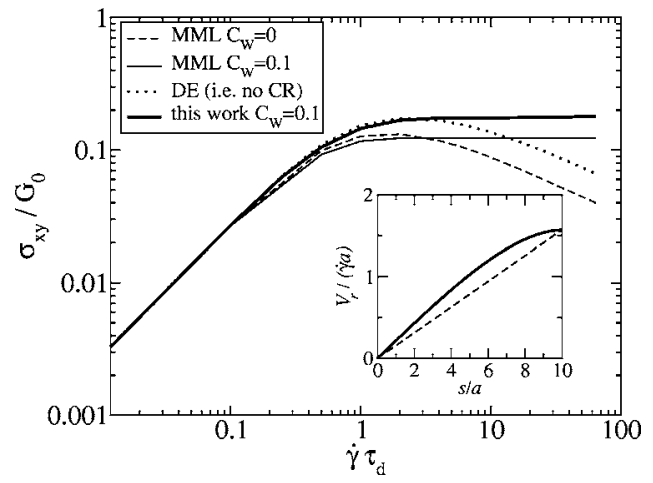

FIG. 3. Steady-state predictions for a chain of 20 segments [(MML $\left.C_{W}=0\right)$ and (MML $\left.\left.C_{W}=0.1\right)\right]$ of the MML model [13] solved in real space without CLF for the values of the $C_{W}$ coefficient equal to 0 and 0.1 , respectively; (DE) of the Doi-Edwards model and the present work for $C_{W}=0$; and (this work $C_{W}=0.1$ ) of the present work without CLF for $C_{W}=0.1$. Inset: dimensionless retraction velocity for $Z=20, \dot{\gamma} \tau_{\mathrm{d}}=1$ as a function of the segment position along the chain; $s=0$ corresponds to the center of the chain. Solid line: retraction speed used in the present work. Dashed line: if an assumption about linear dependence of $V_{r}$ is made [13].

the direction of the flow $x$ and so the $x$ components of the tangent vectors at two different points along the chain cannot be treated independently anymore. Additionally, we would like to point out that the same flaw is expected to be present in any theory that makes use of the assumption of a negligibly small correlation length in a fast, or even moderate, flow.

In Fig. 3 we compare the results of our approach to the Doi-Edwards [2] and MML [13] models. For this purpose we consider the predictions of all the models with $\left(C_{W}=0.1\right)$ or without $\left(C_{W}=0\right)$ constraint release and in the absence of $C L F$. We want to emphasize that $C_{W}=0$ in our theory yields exactly the DE model. This is not surprising: as has been shown in Sec. III A, in the absence of CR, Eq. (11) for the probability distribution function $F_{1}$ coincides with the one by Doi and Edwards [2]. In contrast, in the original work by Milner et al. [13] the DE limit for $C_{W}=0$ is reproduced only qualitatively: there is a maximum in the stress-rate curve but it shows up at too high rates-nearly at $100 / \tau_{\mathrm{d}}$ (see Fig. 3 in [13]). As has been claimed in [14], this artifact is due to the incorrect treatment of the $\delta$ functions and the solution in the Fourier space used in $[12,13]$. Apart from that, additional error is brought by an oversimplified treatment of the retraction rate (16): we remind that Milner et al. [13] assumed it to be constant along the chain. To test the consequences of this assumption alone, we solve the MML model in real space for $C_{W}=0$ and $C_{W}=0.1$, Fig. 3. As is seen from Fig. 3, the wrong position of the maximum in $[12,13]$ indeed results from the incorrect solution in Fourier space. However, the values of the stress predicted by the real-space solution in the nonlinear regime still differ by a factor of 2 from the ones by $\mathrm{DE}$ [compare the curves (DE) and (MML $\left.\left.C_{W}=0\right)\right]$ and the present work [compare (this work) and (MML $\left.C_{W}=0.1\right)$ ]. This discrepancy must be attributed to the oversimplification in the treatment of the retraction rate. To illustrate that, we

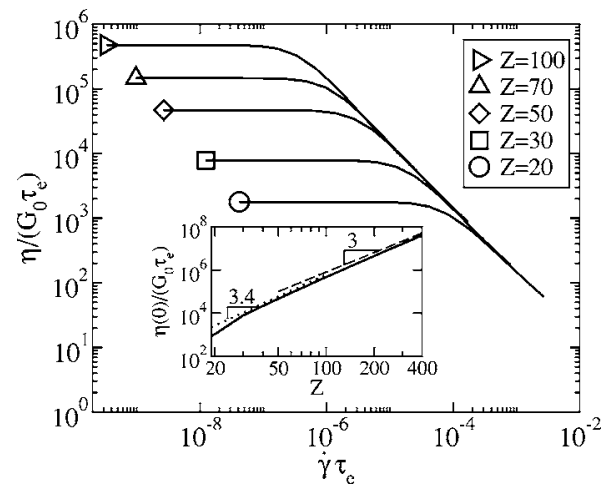

FIG. 4. Steady state shear viscosity $\eta$ vs shear rate $\dot{\gamma}$ (nondimentionalized by $\tau_{\mathrm{e}}$-the Rouse time of one entanglement segment) for $C_{W}=0.1$ and $Z=20,30,50,70,100$. Inset: the zero-shear viscosity scales as $\eta(0) \sim Z^{3.4}$ for $Z \lesssim 200$ and as $\eta(0) \sim Z^{3}$ for $Z$ $\gtrsim 200$.

note that the retraction velocity of the segment $s$ in the absence of CR is given by [2]

$$
V_{r}(s, t)=\int_{0}^{s} \mathrm{~d} x \boldsymbol{K}: \boldsymbol{S}(x, t),
$$

which simplifies to $V_{r}(s)=\dot{\gamma} \int_{0}^{s} \mathrm{~d} x S_{x y}(x)$ for a steady shear flow. A typical $V_{r}(s)$ curve, presented in the inset to Fig. 3, deviates quite significantly from the linear one assumed in [13]. Hence, a general conclusion can be drawn: the unnecessary assumption about a constant retraction rate leads to an error in the predictions of the stress at the shear rates where the plateau region starts.

Finally, we address briefly the chain length dependence of the steady-state viscosity, Fig. 4. For this purpose the time is measured in units of $\tau_{\mathrm{e}}$-the Rouse time of one entanglement strand-which does not depend on $Z$. The zero shear rate viscosity $\eta(0)$ scales as $Z^{3.4}$ for relatively short chains. This effect is achieved because of CLF which speeds up the relaxation of relatively short chains quite considerably. The $\eta(0) \sim Z^{3}$ behavior is approached asymptotically for chains longer than 200 segment - a behavior also predicted in $[5,6]$ and other works.

b. Correlations along the chain under flow conditions.

Besides the purely rheological properties addressed above, the formalism allows us to check explicitly when the correlations should be taken into account. As we have shown before, Fig. 2(b), even relatively large-scale rheological properties are sensitive to the assumption of negligible correlations. In what follows, we aim to test directly the influence of the flow on the correlation length along the polymer.

Strictly speaking, it is impossible to define a correlation length for a finite object rigorously: due to boundary effects, the correlators of the type $\left\langle\hat{\boldsymbol{u}}\left(s_{1}, t\right) \cdot \hat{\boldsymbol{u}}\left(s_{2}, t\right)\right\rangle$ will depend not only on $\left|s_{1}-s_{2}\right|$ but also on $s_{1}$ and $s_{2}$. Therefore, we address the problem semiquantitatively and define the correlation length $a_{c}$ as a decay length of the correlator $\langle\hat{\boldsymbol{u}}(0, t) \cdot \hat{\boldsymbol{u}}(s, t)\rangle$ 


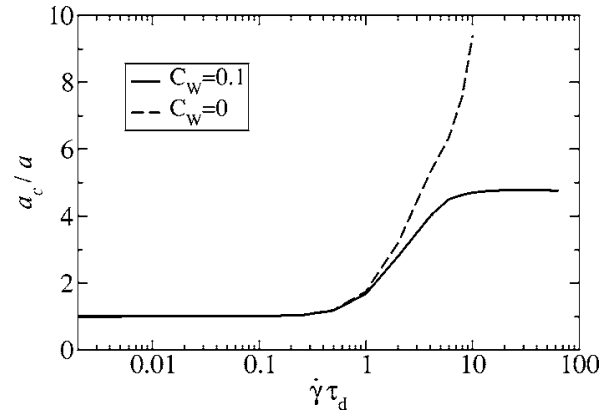

FIG. 5. The correlation length $a_{c}$ with (solid line) and without (dashed line) $\mathrm{CR}$ as a function of the flow rate, $Z=20$.

upon steady-flow conditions. Apparently, because of Eq. (32), $a_{c} \equiv a$ at the equilibrium, as expected. The evolution of $a_{c}$ in the presence of flow is shown in Fig. 5 .

As seen from Fig. 5, for relatively slow flows there is essentially no difference between $a_{c}$ with or without CCR$a_{c} \simeq a$ and chain segments can be treated as being independent. However, in the "moderate-flow" regime $\dot{\gamma} \geqslant \tau_{\mathrm{d}}^{-1}$, where CCR is typically active, correlations for $C_{W}=0$ grow much more rapidly. It supports the fact that in the DE model the chains become completely aligned with the flow. Constraint release changes the picture considerably-there are still correlations present, but $a_{c}$ is several times smaller than without CR. The above analysis leads to conclusions about the validity regions of the models. E.g., the DE theory [2], which makes explicit use of the assumption about a small correlation length, is not valid for the $\dot{\gamma}>1 / \tau_{\mathrm{d}}$ regime. Also approximations of the type (41) are, strictly speaking, not valid there-therefore the difference between the stress curves shown in Fig. 2(b) is not surprising. In general, Fig. 5 signals that for $\dot{\gamma} \tau_{\mathrm{d}}>1$ correlations along the chain have to be taken into account and approximations ignoring this fact are doubtful.

\section{CONCLUDING REMARKS}

In this paper we presented a framework allowing for a consistent description of the correlation effects along a poly- mer chain under flow conditions. The formalism is based on the many-point probability distribution function $F_{n}$. The equation of motion for $F_{n}$ takes into account such relaxation mechanisms as reptation, retraction, constraint release, and contour length fluctuation.

It has been shown that some known models can be derived from the general formalism and correspond to certain closure approximations. E.g., the simplest one (28) gives the Doi-Edwards equation for $F_{1}$ without independent alignment [2]. A somewhat more sophisticated closure (30) corresponds to an improved version (31) of the MML theory [13]. In principle, more complicated closure relations can be used to take into account three- and more-point correlation functions.

One of the main goals pursued in this work is the analysis of the consequences of different assumptions widely used in the literature but rarely supported by rigorous calculations. Comparing the one- and two-point approximations, we show that some rheological functions $\left(\sigma_{x y}\right.$, steady-state viscosity, etc.) are quite insensitive to the assumption about small correlation lengths in the presence of flow, whereas others (normal stress difference, extinction angle, etc.) show a clear sensitivity on this point. Also, a direct estimation of the correlation length shows that it cannot be neglected in moderate- or fast-flow regimes.

Apparently, the model proposed is not free of simplifying assumptions. Apart from the shortcomings common to all tube-based models, the absence of the stretch restricts the model's applicability to flows with rates up to the inverse Rouse time. However, since for long polymers the Rouse time is much smaller than $\tau_{\mathrm{d}}$, the model is still applicable to the most interesting flow regimes. Unfortunately, fast elongational flows, where chain stretch plays a key role, are thus outside the scope of the approach. It still remains a challenge to incorporate stretch effects into the proposed formalism.

\section{ACKNOWLEDGMENTS}

This research is supported by the Technology Foundation STW, applied science division of NWO, and the technology programme of the Ministry of Economic Affairs of The Netherlands.
[1] J. D. Ferry, Viscoelastic Properties of Polymers (Wiley, New York, 1970).

[2] M. Doi and S. Edwards, The Theory of Polymer Dynamics (Clarendon Press, Oxford, 1986).

[3] P. G. de Gennes, Scaling Concepts in Polymer Physics (Cornell University Press, Ithaca, 1985).

[4] P. G. de Gennes, J. Chem. Phys. 55, 572 (1971).

[5] M. Doi, J. Polym. Sci., Polym. Phys. Ed. 21, 667 (1983).

[6] S. T. Milner and T. C. B. McLeish, Phys. Rev. Lett. 81, 725 (1998).

[7] G. Marrucci and N. Grizzuti, Gazz. Chim. Ital. 118, 179 (1988).

[8] G. Marrucci, J. Non-Newtonian Fluid Mech. 62, 279 (1996).
[9] G. Ianniruberto and G. Marrucci, J. Non-Newtonian Fluid Mech. 65, 241 (1996).

[10] G. Ianniruberto and G. Marrucci, J. Non-Newtonian Fluid Mech. 102, 383 (2002).

[11] D. W. Mead, R. G. Larson, and M. Doi, Macromolecules 31, 7895 (1998).

[12] A. E. Likhtman, S. T. Milner, and T. C. B. McLeish, Phys. Rev. Lett. 85, 4550 (2000).

[13] S. T. Milner, T. C. B. McLeish, and A. E. Likhtman, J. Rheol. 45, 539 (2001).

[14] R. S. Graham, A. E. Likhtman, T. C. B. McLeish, and S. T. Milner, J. Rheol. 47, 1171 (2003).

[15] M. A. Tchesnokov, J. Molenaar, J. J. M. Slot, and R. Stepan- 
yan, J. Non-Newtonian Fluid Mech. 123, 185 (2004).

[16] R. Stepanyan, J. J. M. Slot, and J. Molenaar, Europhys. Lett. 68, 832 (2004).

[17] J. Bent et al., Science 301, 1691 (2003).

[18] I. Z. Fisher, The Statistical Theory of Liquids (University of Chicago Press, Chicago, 1964).

[19] S. T. Milner and T. C. B. McLeish, Macromolecules 31, 7479 (1998).

[20] J. L. Viovy, M. Rubinstein, and R. H. Colby, Macromolecules
24, 3587 (1991).

[21] A. E. Likhtman and R. S. Graham, J. Non-Newtonian Fluid Mech. 114, 1 (2003).

[22] R. B. Bird, C. F. Curtiss, R. C. Armstrong, and O. Hassager, Dynamics of Polymeric Liquids (Wiley, New York, 1987), Vol. 2.

[23] G. H. Golub, and J. M. Ortega, Scientific Computing and Differential Equations (Academic Press, San Diego, 1992).

[24] M. Doi, J. Polym. Sci., Polym. Phys. Ed. 18, 2055 (1980). 